\title{
A NEW PLANET AROUND AN M DWARF: REVEALING A CORRELATION BETWEEN EXOPLANETS AND STELLAR MASS ${ }^{1}$
}

\author{
John Asher Johnson, ${ }^{2}$ R. Paul Butler ${ }^{3}$ Geoffrey W. Marcy, ${ }^{2}$ Debra A. Fischer, ${ }^{4}$ \\ Steven S. Vogt, ${ }^{5}$ Jason T. Wright, ${ }^{2}$ and Kathryn M. G. Peek $^{2}$ \\ Received 2007 June 15; accepted 2007 July 17
}

\begin{abstract}
We report precise Doppler measurements of GJ 317 (M3.5 V) that reveal the presence of a planet with a minimum mass $M_{P} \sin i=1.2 M_{\text {Jup }}$ in an eccentric, 692.9 day orbit. GJ 317 is only the third M dwarf with a Doppler-detected Jovian planet. The residuals to a single-Keplerian fit show evidence of a possible second orbital companion. The inclusion of a second Jupiter-mass planet $\left(P \approx 2700\right.$ days, $\left.M_{P} \sin i=0.83 M_{\text {Jup }}\right)$ decreases $\sqrt{\chi_{\nu}^{2}}$ from 2.02 to 1.23 , and reduces the rms from 12.5 to $6.32 \mathrm{~m} \mathrm{~s}^{-1}$. A false-alarm test yields a $1.1 \%$ probability that the curvature in the residuals of the single-planet fit is due to random fluctuations, lending additional credibility to the two-planet model. However, our data only marginally constrain a two-planet fit, and further monitoring is necessary to fully characterize the properties of the second companion. To study the effect of stellar mass on giant planet occurrence, we measure the fraction of stars with planets in three mass bins comprised of our samples of M Dwarfs, solar-mass stars, and intermediate-mass subgiants. We find a positive correlation between stellar mass and the occurrence rate of Jovian planets within $2.5 \mathrm{AU}$. Low-mass $\mathrm{K}$ and $\mathrm{M}$ stars have a $1.8 \% \pm 1.0 \%$ planet occurrence rate compared to $4.2 \% \pm 0.7 \%$ for solar-mass stars and $8.9 \% \pm 2.9 \%$ for the higher mass subgiants. This result indicates that the former F- and A-type stars with $M_{*} \geq 1.3 M_{\odot}$ in our sample are nearly 5 times more likely than the $\mathrm{M}$ dwarfs to harbor a giant planet. Our analysis shows that the correlation between Jovian planet occurrence and stellar mass exists even after correcting for the effects of stellar metallicity.
\end{abstract}

Subject headings: planetary systems: formation — stars: individual (GJ 317) — techniques: radial velocities

\section{INTRODUCTION}

A planet host star inherits its fundamental characteristics from the same disk material that forms its planets. Studying the relationships between the observed occurrence rate of giant planets as a function of the characteristics of their host stars therefore provides crucial tests of planet formation theories. The two most fundamental properties of stars are mass and chemical composition. A number of detailed spectroscopic analyses of nearby stars have revealed a strong correlation between the metallicity of stars and the likelihood that they harbor detectable planets (Gonzalez 1997; Santos et al. 2004; Fischer \& Valenti 2005). This finding can be understood in the context of the core accretion model: increasing the metallicity of star/disk system increases the surface density of solid particulate matter, which leads to an enhanced growth rate for protoplanetary cores (Ida \& Lin 2004, 2005a; Kornet et al. 2005).

Another way to enhance the surface density of solid material in the midplane of the protoplanetary disk is to increase the total mass of the disk (Ida \& Lin 2005b). If disk masses scale with stellar mass, then there should be an observed correlation between planet occurrence and stellar mass. The relationship between stellar mass and planet formation rates has been explored theoretically in the context of the core accretion model. Laughlin et al. (2004) simulated the formation of planets in disks around low-mass stars and found that the lower surface densities and longer orbital

\footnotetext{
${ }^{1}$ Based on observations obtained at the W. M. Keck Observatory, which is operated jointly by the University of California and the California Institute of Technology. Keck time has been granted by both NASA and the University of California.

2 Department of Astronomy, University of California, Berkeley, CA 94720.

3 Department of Terrestrial Magnetism, Carnegie Institution of Washington, 5241 Broad Branch Road NW, Washington, DC 20015-1305.

${ }_{4}^{4}$ Department of Physics and Astronomy, San Francisco State University, San Francisco, CA 94132.

${ }_{5}^{5}$ UCO/Lick Observatory, University of California, Santa Cruz, CA 95064.
}

timescales in the disks around $\mathrm{M}$ dwarfs impede the growth of Jupiter-mass planets. By the time the critical core mass is reached $\left(\approx 10 M_{\oplus}\right)$, the supply of disk gas is exhausted due to accretion onto the central star and photoevaporation. Laughlin et al. (2004) therefore predict an abundance of "failed" gas giants with masses comparable to Neptune and a much lower frequency of Jupitermass planets. Ida \& Lin (2005b) find very similar results from their simulations of planet formation around stars of various masses.

The mass of the central star also influences the radial extent of the region in which protoplanetary cores form. Kennedy \& Kenyon (2007) account for disk accretion and the evolving luminosity of the central star to model the influence of stellar mass on the location and size of the core-forming region in circumstellar disks. Similar to Laughlin et al. (2004) and Ida \& Lin (2005b) they predict that M dwarfs should exhibit a deficit of giant planets. Kennedy \& Kenyon (2007) also predict that the fraction of stars with Jovian planets should increase with stellar mass up to a peak near $M_{*} \approx 3 M_{\odot}$.

The theoretical prediction of a lower frequency of Jupiter-mass planets around low-mass $\mathrm{M}$ dwarfs is in agreement with the current observational data. About 300 nearby $\mathrm{M}$ dwarfs are currently monitored by various Doppler surveys, and only five have been discovered to harbor one or more planets (Delfosse et al. 1998; Marcy et al. 1998, 2001; Endl et al. 2003; Bonfils et al. 2005b, 2007; Butler et al. 2006b). Three additional planets have been discovered orbiting distant $(\sim 1 \mathrm{kpc})$ low-mass stars by gravitational lensing surveys (Bond et al. 2004; Gould et al. 2006; Beaulieu et al. 2006; Bennett et al. 2006).

The majority of the Doppler-detected planets around M dwarfs are significantly smaller than Jupiter, with minimum masses $\left(M_{P} \sin i\right)$ comparable to the masses of Neptune and Uranus (5$23 M_{\oplus}$ ). The sample of low-mass, Doppler-detected planets consists of the $22.6 \pm 1.9 M_{\oplus}$ planet around GJ 436 (Butler et al. 2004; Maness et al. 2007); the inner, $M_{P} \sin i=5.9 M_{\oplus}$ planet around GJ 876 (Rivera et al. 2005); the $M_{P} \sin i=11 M_{\oplus}$ planet 
orbiting GJ 674 (Bonfils et al. 2007); and the triple system orbiting GJ 581, which consists of planets with minimum masses 16.6, 5, and $8 M_{\oplus}$ (Bonfils et al. 2005b; Udry et al. 2007). The kinship between these extrasolar "super-Earths" and the ice giants in our solar system was confirmed with the recent discovery that the Neptune-mass planet around GJ 436 transits its central star (Gillon et al. 2007). The transit light curve provides an absolute measurement of the planet's mass and radius, suggesting an internal structure very much like Neptune, with a rocky core likely surrounded by a thick water layer and thin gaseous envelope (Fortney et al. 2007).

To date, there are only two nearby M dwarfs known to harbor Jupiter-mass companions: GJ 876 (Marcy et al. 1998, 2001; Rivera et al. 2005) and GJ 849 (Butler et al. 2006a). The relatively small number of Jovian planets around M dwarfs is not due to decreased Doppler sensitivity. For a planet of a given mass and orbital period, the amplitude of a host star's reflex velocity scales as $K \propto M_{*}^{-2 / 3}$, making planets easier to detect around stars with lower masses. Butler et al. (2006a) showed that the frequency of giant planets is 2-3 times higher among solar-mass stars compared to $\mathrm{M}$ dwarfs (see also Laws et al. 2003; Endl et al. 2006; Bonfils et al. 2007). However, the uncertainty in the estimated planet occurrence rate for $\mathrm{M}$ dwarfs is large due to the small number of target stars and planet detections.

Another obstacle that has so far hindered a study of the effects of stellar mass on planet formation is the limited range of masses spanned by Doppler-based planet searches. For example, the solarmass FGK dwarfs that comprise the bulk of the California and Carnegie Planet Search (CCPS) span stellar masses from 0.8 to 1.2 $M_{\odot}$ (Valenti \& Fischer 2005; Takeda et al. 2007). Much more leverage can be gained by measuring the planet occurrence rate around higher mass F- and A-type stars $\left(1.3 M_{\odot} \leq M_{*} \lesssim 3.0 M_{\odot}\right)$. Unfortunately, intermediate-mass, main-sequence stars are poor precision Doppler targets. Stars with spectral types earlier than F8 tend to have rotationally broadened absorption features (do Nascimento et al. 2003; Galland et al. 2005), have fewer spectral lines due to high surface temperatures, and display a large amount of excess velocity scatter due to surface inhomogeneities and pulsation ("jitter"; Saar et al. 1998; Wright 2005).

We have addressed the limited range of stellar masses in Dopplerbased planet searches by conducting a search for planets around stars with masses bracketing those of the Sun-like stars in the main CCPS sample. At higher stellar masses we are conducting a search for planets around intermediate-mass subgiants at the Lick and Keck Observatories (Johnson et al. 2006a, 2007). Subgiants are evolved stars that have cooler surface temperatures and lower rotational velocities than their A- and F-type main-sequence progenitors $\left(T_{\text {eff }} \approx 5000 \mathrm{~K}\right.$ compared to $T_{\text {eff }}>6000 \mathrm{~K}$, and $V_{\text {rot }} \sin i<5$ vs. $V_{\text {rot }} \sin i \gg 50 \mathrm{~km} \mathrm{~s}^{-1}$, respectively). The spectra of subgiants therefore have an increased number of narrow absorption lines required for precision Doppler measurements, making them ideal proxies for A- and F-type main-sequence stars.

At the low-mass end we have been monitoring a sample of 147 late-K through $\mathrm{M}$ dwarfs $\left(M_{*} \lesssim 0.6 M_{\odot}\right)$ as part of the NASA Keck M Dwarf Planet Survey (Butler et al. 2006b; Rauscher \& Marcy 2006). The 7 year baseline of our M dwarfs survey, together with the 3 year duration of our subgiants planet search, now provide an excellent opportunity to measure the relationship between stellar mass and planet occurrence for stars with a wide range of masses and comparable detection characteristics. Here, we report the detection of a Jupiter-mass planet in a $1.897 \mathrm{yr}$ orbit around the M3.5 dwarf GJ 317. We present the stellar characteristics of the host star in $\S 2$. In $\S 3$ we present our observations and orbit solution, including an assessment of a possible second
Jovian companion in the system. In $\S 4$ we incorporate this latest $\mathrm{M}$ dwarf planet detection into a detailed analysis of the relationship between stellar mass and the occurrence rate of giant planets. We conclude in $\S 5$ with a summary and a brief discussion of our results.

\section{PROPERTIES OF GJ 317}

GJ 317 (LHS 2037, L675-081) is a nearby M3.5 dwarf among the 147 low-mass stars in the NASA Keck M Dwarf Planet Survey (Rauscher \& Marcy 2006). The Simbad database lists an apparent magnitude $V=13.0$. However, this magnitude is $\sim 1$ mag fainter than the values listed in several other catalogs: the Gliese-Jahreiss Catalog (GJ Catalog; Gliese \& Jahreiss 1991) gives $V=12.0$, the Carlsberg Meridian Catalogs list $V=12.03$, and the Catalogue of Stellar Spectral Classifications lists $V=11.98 .{ }^{6}$ We adopt the mean of these measurements, $V=12.0$. The GJ Catalog also lists $B-$ $V=1.52$ and a trigonometric parallax $\pi=101.3 \pm 27$ mas. The GJ Catalog color agrees well with the color measured by Reid et al. (2002; $B-V=1.53)$, and the listed parallax is consistent with the value measured by Woolley $(1970 ; \pi=116 \pm 12$ mas $)$. We adopt the mean of the two trigonometric parallax measurements, $\pi=109.0 \pm 20.0$ mas. This value is $1 \sigma$ larger (based on the quadrature sum of the uncertainties) than the "resulting parallax" $\pi=$ $87 \pm 17$ mas listed in the GJ Catalog, which is estimated from the star's broadband colors and spectral type. Our adopted trigonometric parallax yields a distance of $9.17 \pm 1.7 \mathrm{pc}$ and an absolute visual magnitude $M_{V}=12.2$.

For its $B-V$ color GJ 317 is unusually faint, lying $\approx 1.8$ mag below the mean Hipparcos main sequence as defined by Wright (2004). Its location in the H-R diagram suggests that GJ 317 is an extremely metal-poor subdwarf. However, the star's Two Micron All Sky Survey (2MASS) infrared magnitudes together with the $K$-band photometric metallicity-luminosity calibration of Bonfils et al. (2005a) suggest that GJ 317 has a metallicity consistent with solar, $[\mathrm{Fe} / \mathrm{H}]=-0.23 \pm 0.2$. This metallicity is much higher than the value expected given the star's position in the H-R diagram.

We compared our iodine-free template spectrum of GJ 317 to a template spectrum of a similar M dwarf, GJ 849 (M3.5, $V=$ $10.4, B-V=1.52,[\mathrm{Fe} / \mathrm{H}]=+0.16 \pm 0.2$; Butler et al. 2006a). Figure 1 shows a comparison of a small portion of the template spectra, which shows that these two stars are remarkably similar. Since the line depths are nearly identical we can safely conclude that GJ 317 is not a metal-deficient subdwarf. As an additional check, we compared the 2MASS $J-K$ and $H-K$ colors of GJ 317 to those of GJ 849. The infrared colors of the two stars agree to within 0.001 and $0.006 \mathrm{mag}$, respectively, again suggesting very similar spectral characteristics.

We can imagine several possible explanations for the abnormal location of GJ 317 in the H-R diagram. The first possibility is that the parallaxes listed in the literature are systematically incorrect, and GJ 317 is actually significantly farther away than the reported parallaxes suggest. Similarly, the star's reported apparent $V$ magnitude may be too faint. Another possibility is that the star's $B-V$ color is too blue by $\sim 0.1 \mathrm{mag}$. Finally, the star may be obscured by 1.5 mag of gray extinction. We are planning follow-up photometric monitoring during the next observing season to further investigate the latter three scenarios.

We used the 2MASS infrared photometry of GJ 317, together with the $K$-band mass-luminosity calibration of Delfosse et al. (2000), to estimate a stellar mass $M_{*}=0.26 \pm 0.04 M_{\odot}$. This mass estimate agrees well with the $0.22 \pm 0.05 M_{\odot}$ value predicted by the $V$-band mass-luminosity relationship of Bonfils et al. (2005a).

\footnotetext{
${ }^{6}$ Vizier Online Data Catalog, 3233 (A. B. Skiff, 2004).
} 


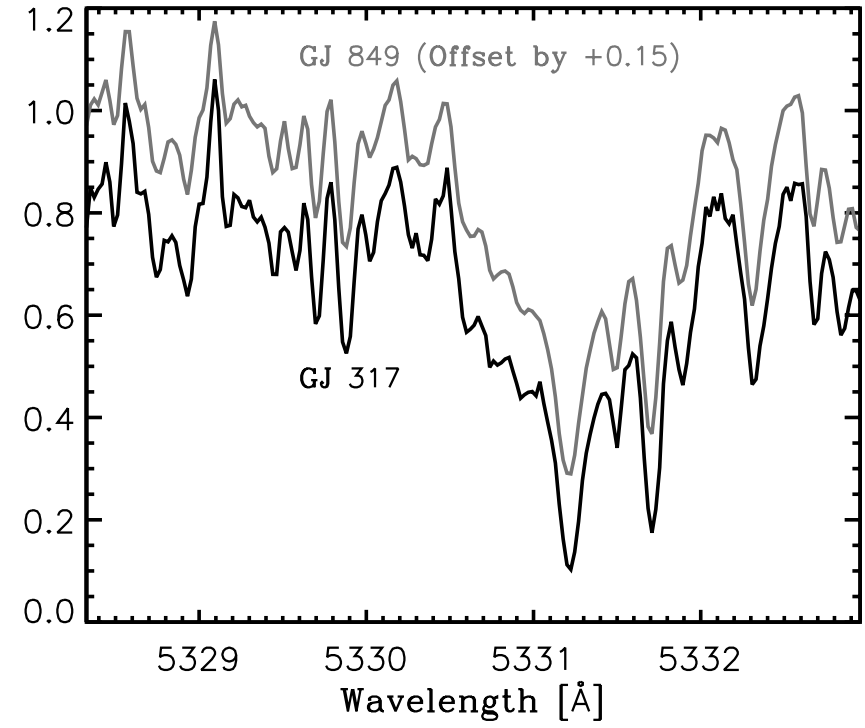

FIG. 1.-Comparison of the spectra of GJ 317 (black line) and GJ 849 ( gray line), which has a similar $B-V$ color, spectral type, and metallicity $([\mathrm{Fe} / \mathrm{H}]=+0.16 \pm$ 0.2 ). The spectrum of GJ 849 has been shifted up by 0.15 for clarity. Since the spectral lines of the two stars have very similar depths, we conclude that GJ 317 cannot have a metallicity much different than that of GJ 849. GJ 317 is therefore not a metal-poor subdwarf, despite its apparent location 2 mag below the mean Hipparcos main sequence.

We adopt the mean of these two estimates, $M_{*}=0.24 M_{\odot}$. The properties of GJ 317 are summarized in Table 2.

\section{OBSERVATIONS AND ORBITAL SOLUTIONS}

We have been monitoring GJ 317 with the Keck I 10 m telescope for 7.4 years. We obtained high-resolution spectra using the HIRES echelle spectrometer (Vogt et al. 1994) with an iodine cell mounted directly in front of the entrance slit. The dense set of molecular absorption lines provide a robust wavelength fiducial, as well as information about the shape of the spectrometer instrumental profile (Marcy \& Butler 1992; Valenti et al. 1995). The Doppler shift is measured from each star-plus-iodine observation using the modeling procedure described by Butler et al.

TABLE 1

Radial Velocities for GJ 317

\begin{tabular}{|c|c|c|}
\hline $\begin{array}{c}\mathrm{JD} \\
(-2,440,000)\end{array}$ & $\begin{array}{c}\mathrm{RV} \\
\left(\mathrm{m} \mathrm{s}^{-1}\right)\end{array}$ & $\begin{array}{c}\text { Uncertainty } \\
\left(\mathrm{m} \mathrm{s}^{-1}\right)\end{array}$ \\
\hline $11,550.993 \ldots$ & 13.89 & 3.79 \\
\hline $11,552.990 \ldots$ & 30.27 & 4.99 \\
\hline $11,582.891 \ldots$. & 45.77 & 4.16 \\
\hline $11,883.101 \ldots \ldots \ldots \ldots \ldots$ & -23.35 & 3.74 \\
\hline $11,973.795 \ldots \ldots \ldots \ldots \ldots \ldots$ & -65.89 & 6.10 \\
\hline $12,243.073 \ldots \ldots \ldots \ldots \ldots \ldots$ & 0.00 & 6.52 \\
\hline $12,362.949 \ldots \ldots \ldots \ldots \ldots$ & 95.56 & 6.07 \\
\hline $12,601.045 \ldots \ldots \ldots \ldots \ldots \ldots$ & -31.64 & 5.09 \\
\hline $12,989.125 \ldots \ldots \ldots \ldots \ldots$ & 101.25 & 4.79 \\
\hline $13,369.016 \ldots \ldots \ldots \ldots \ldots$ & -29.65 & 3.40 \\
\hline $13,753.983 \ldots \ldots \ldots \ldots \ldots$ & 130.31 & 3.68 \\
\hline 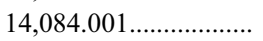 & -6.55 & 4.64 \\
\hline $14,086.141 \ldots \ldots \ldots \ldots \ldots . .$. & -21.73 & 4.55 \\
\hline $14,130.082 \ldots \ldots \ldots \ldots \ldots$ & -15.26 & 4.79 \\
\hline $14,131.014 \ldots \ldots \ldots \ldots \ldots$ & -15.47 & 4.20 \\
\hline $14,138.932 \ldots \ldots \ldots \ldots \ldots$ & -8.98 & 2.82 \\
\hline $14,216.733 \ldots \ldots \ldots \ldots \ldots \ldots$ & 4.15 & 3.86 \\
\hline $14,255.745 \ldots \ldots \ldots \ldots \ldots \ldots$ & 28.54 & 2.25 \\
\hline
\end{tabular}

TABLE 2

Stellar Properties for GJ 317

\begin{tabular}{|c|c|}
\hline Parameter & Value \\
\hline$V .$. & 12.0 \\
\hline$B-V$ & 1.52 \\
\hline$M_{V}$ & 12.2 \\
\hline 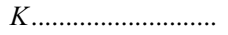 & 7.016 \\
\hline$J-K \ldots \ldots \ldots \ldots \ldots \ldots \ldots$ & 0.915 \\
\hline$H-K$ & 0.311 \\
\hline$d(\mathrm{pc}) \ldots \ldots \ldots \ldots \ldots \ldots \ldots$ & $9.17 \pm 1.7$ \\
\hline$M_{*}\left(M_{\odot}\right) \ldots \ldots \ldots \ldots \ldots$ & $0.24 \pm 0.04$ \\
\hline$[\mathrm{Fe} / \mathrm{H}] \ldots \ldots \ldots \ldots \ldots$ & $-0.23 \pm 0.2$ \\
\hline
\end{tabular}

(1996). Figure 2 shows velocity measurements of four stable M Dwarfs, demonstrating our long-term Doppler precision of $3-4 \mathrm{~m} \mathrm{~s}^{-1}$.

A total of 18 Doppler measurements of GJ 317 are listed in Table 1 and shown in Figure 3. We carried out a search for the best-fit Keplerian orbital solution using a nonlinear, least-squares algorithm. In the fitting procedure, each velocity measurement is assigned a weight constructed from the quadrature sum of the internal measurement uncertainty and a stellar jitter term. The internal uncertainty is the weighted standard deviation of the mean velocity measured from each of the $\sim 7002 \AA$ chunks in each echelle spectrum. The stellar jitter term accounts for noise in excess

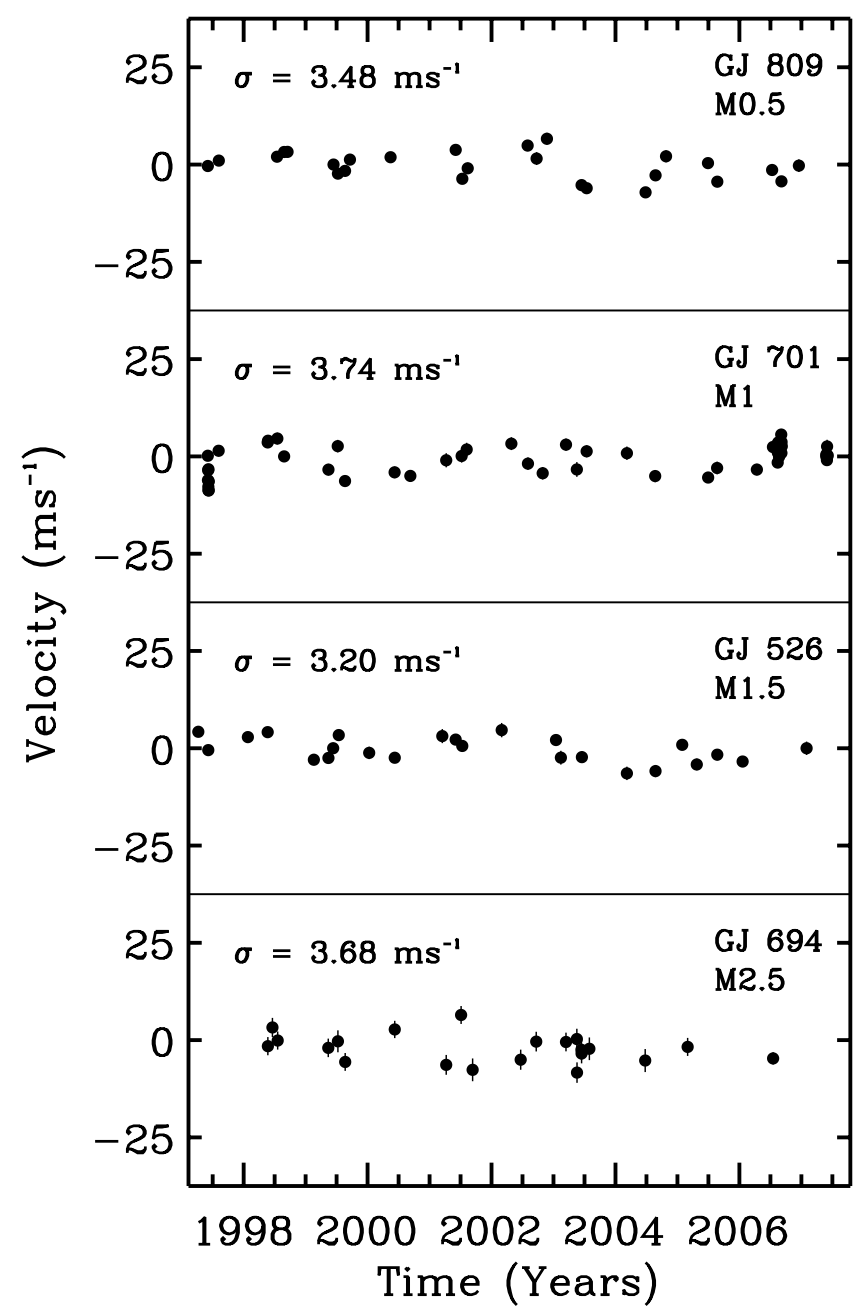

FIG. 2.- Radial velocity time series for four stable $\mathrm{M}$ dwarfs in our Keck Doppler survey demonstrating a Doppler precision of $3-4 \mathrm{~m} \mathrm{~s}^{-1}$ over the past $8-9 \mathrm{yr}$. 


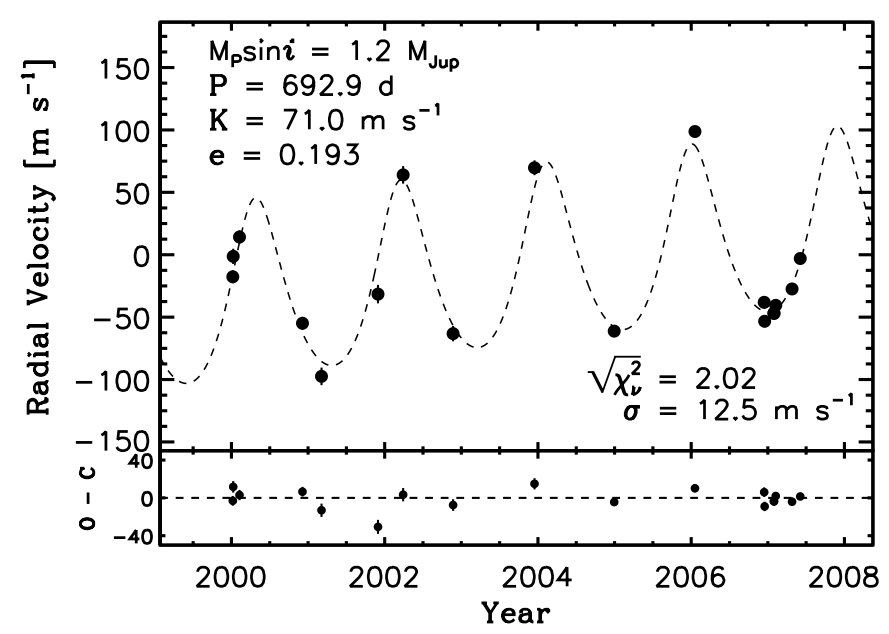

FIG. 3.-Our radial velocity time series for GJ 317, based on observations from Keck Observatory. The dashed line shows the best-fit Keplerian with an additional linear trend of $7.6 \pm 1 \mathrm{~m} \mathrm{~s}^{-1} \mathrm{yr}^{-1}$. The rms scatter of the residuals (bottom) is much larger than the predicted $6 \mathrm{~m} \mathrm{~s}^{-1}$ of scatter due to measurement uncertainties and stellar jitter.

of the internal uncertainties due to astrophysical sources such as rotational modulation of stellar surface features and stellar pulsation (Saar et al. 1998; Wright 2005). For Sun-like dwarfs the stellar jitter can be accurately predicted based on the chromospheric diagnostic $\log R_{\mathrm{HK}}^{\prime}$, given by the ratio of the $\mathrm{Ca}$ II $\mathrm{H}$ and $\mathrm{K}$ flux to the stellar UV continuum, along with luminosity and $B-V$ color. However, $\log R_{\mathrm{HK}}^{\prime}$ is not calibrated for $\mathrm{M}$ dwarfs because stars with such cool atmospheres lack a well-defined continuum due to molecular line blanketing. We therefore estimate the stellar jitter of the $\mathrm{M}$ dwarfs in our sample by assuming a constant value for all stars with $B-V>1.3$. We selected the $133 \mathrm{M}$ stars in our sample with 12 or more observations and measured the jitter using the prescription of Wright (2005). For HIRES observations made prior to the 2004 CCD upgrade we derive a median jitter of $3.5 \mathrm{~m} \mathrm{~s}^{-1}$, and $2.0 \mathrm{~m} \mathrm{~s}^{-1}$ for post-upgrade observations.

Our search for a best-fitting Keplerian to the 18 observations of GJ 317 reveals a minimum in $\sqrt{\chi_{\nu}^{2}}$ for an orbital period near $P=690$ days. However, the rms residual to this best-fitting, singleplanet model is $16.8 \mathrm{~m} \mathrm{~s}^{-1}$, which is much higher than the expected scatter of $\approx 6 \mathrm{~m} \mathrm{~s}^{-1}$ from the internal errors and jitter. The resulting reduced $\sqrt{\chi_{\nu}^{2}}=2.67$ indicates the single-planet model is inadequate.

We attempted to improve on the single-Keplerian model by adding a variable linear trend. The existence of a trend in the velocities implies an additional, long-period orbital companion in the system. The dashed line in Figure 3 shows the best-fitting Keplerian with a linear trend of $7.6 \pm 1 \mathrm{~m} \mathrm{~s}^{-1} \mathrm{yr}^{-1}$. The inclusion of a trend reduces the rms from 16.8 to $12.5 \mathrm{~m} \mathrm{~s}^{-1}$, and decreases $\sqrt{\chi_{\nu}^{2}}$ from 2.67 to 2.02, after accounting for the extra free parameter. The Keplerian parameters of this fit are listed in Table 3, along with their estimated uncertainties. The parameter uncertainties were derived using a Monte Carlo method (e.g., Marcy et al. 2005). Our best-fit orbital solution yields a 1.897 yr period, velocity semiamplitude $K=100 \mathrm{~m} \mathrm{~s}^{-1}$, and eccentricity $0.193 \pm 0.06$. Using our adopted stellar mass $M_{*}=0.24 M_{\odot}$, we find a minimum planet mass $M_{P} \sin i=1.2 M_{\mathrm{Jup}}$ and semimajor axis $a=0.95 \mathrm{AU}$.

\subsection{The Two-Planet Model}

Motivated by the success of the Keplerian-plus-trend model, we performed a search for a double-Keplerian fit. The best-fitting,
TABLE 3

Orbital SOlution FOR GJ 317в

\begin{tabular}{|c|c|}
\hline Parameter & Value \\
\hline$P$ (days). & $692.9 \pm 4$ \\
\hline$P(\mathrm{yr}) \ldots \ldots$ & $1.897 \pm 0.01$ \\
\hline$K\left(\mathrm{~m} \mathrm{~s}^{-1}\right) \ldots \ldots \ldots \ldots \ldots$ & $71.0 \pm 7$ \\
\hline е & $0.193 \pm 0.06$ \\
\hline$T_{P}($ Julian date $) \ldots \ldots .$. & $2451639 \pm 30$ \\
\hline Linear trend $\left(\mathrm{m} \mathrm{s}^{-1} \mathrm{yr}^{-1}\right) \ldots \ldots \ldots \ldots \ldots \ldots$ & $7.6 \pm 1$ \\
\hline$\omega(\operatorname{deg})$ & $344 \pm 10$ \\
\hline$M \sin i\left(M_{\mathrm{Jup}}\right)$ & 1.2 \\
\hline 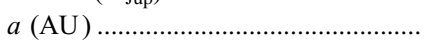 & 0.95 \\
\hline$N_{\text {obs }} \ldots \ldots \ldots \ldots \ldots$ & 18 \\
\hline $\mathrm{rms}\left(\mathrm{m} \mathrm{s}^{-1}\right)$ & 12.5 \\
\hline$\sqrt{\chi_{\nu}^{2}} \ldots \ldots \ldots \ldots$ & 2.02 \\
\hline
\end{tabular}

two-planet model is shown in Figure 4, with orbital periods of 673.4 and 2700 days, respectively. The minimum mass of the inner planet changes from 1.2 to $1.3 M_{\text {Jup }}$, compared the Keplerianplus-trend model. The rms scatter drops from 12.5 to $6.32 \mathrm{~m} \mathrm{~s}^{-1}$ with a resulting decrease in $\sqrt{\chi_{\nu}^{2}}$ from 2.02 to 1.23 .

While the quality of the double-planet fit is encouraging, it is still possible that random noise and jitter could conspire to produce a false curvature in our sparse series of measurements. If this is the case, then the second Keplerian and its associated four additional parameters are an unjustified embellishment on the single-Keplerian-plus-trend model. We tested this null hypothesis by employing the scrambled residuals false-alarm test described by Wright et al. (2007). We first subtract the single-Keplerianplus-trend model from the data and adopt the residuals as random variates to construct a large number of mock velocity time series. Each mock time series is constructed by scrambling the residuals, with replacement, using a pseudorandom number generator. The scrambled residuals are then added back to original data to generate a mock data set. For each mock time series we perform a full search for a best-fit Keplerian-plus-trend orbital solution. If the curvature in the original data is an artifact of random noise, then many of the mock data sets should produce lower $\sqrt{\chi_{\nu}^{2}}$ than the

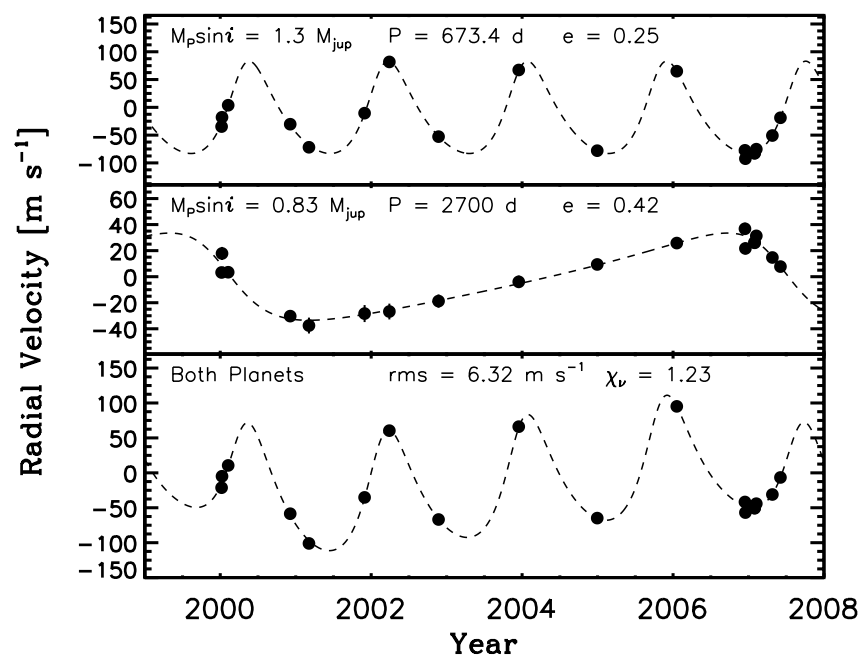

FIG. 4.-Best-fitting two-planet model. The top two panels show the singleplanet fits with the other component removed. The addition of an outer planet with a period $P \approx 2700$ days decreases the rms scatter of the residuals from 12.5 to $6.32 \mathrm{~m} \mathrm{~s}^{-1}$, and the reduced $\sqrt{\chi_{\nu}^{2}}$ from 2.02 to 1.23 . However, the duration and time sampling of our observations only marginally constrain the 11 free parameters of the two-planet fit. 


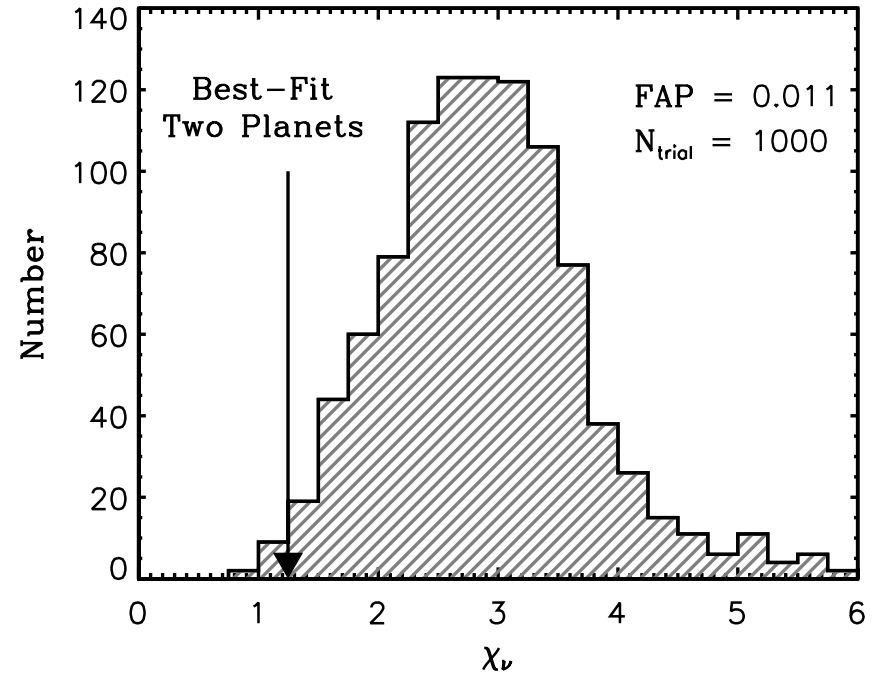

FIG. 5.-Empirical assessment of the FAP of the two-Keplerian model. The observed velocities yield a double-planet orbital solution with $\sqrt{\chi_{\nu}^{2}}=1.11$ (arrow). The histogram shows the distribution of $\sqrt{\chi_{\nu}^{2}}$ obtained from single-Keplerian fits to the mock velocity sets, with a variable linear trend. The mock data sets are generated by adding the scrambled residuals from a single-Keplerian fit to the observed velocities; 11 of the 1000 mock data sets produced orbital fits with $\sqrt{\chi_{\nu}^{2}}$ lower than the original time series, indicating a FAP of 0.011 that random fluctuations are a viable explanation for the quality of the two-Keplerian fit to the observations. Additional observations are required to fully characterize the outer companion and reduce the FAP to below 0.01 .

two-planet fit to the original data. However, if a second, resolved Keplerian signal is present in the observations, then the mock data sets should produce fits that are much worse on average than the original velocities.

The results of our test are shown in Figure 5. We generated 1000 mock data sets and found that only 11 produced $\sqrt{\chi_{\nu}^{2}}$ equal to or lower than the original velocities, resulting in an false-alarm probability $(\mathrm{FAP})=0.011$. The $1.1 \% \mathrm{FAP}$ is promising, but we are nonetheless cautious about the validity of this two-planet model. Given the relatively low amplitude of the second signal and long period of the two candidate planets, our 18 measurements provide only 12 independent data points. The two-planet model is therefore only marginally constrained by our present data. Furthermore, the duration of our observations only covers one orbit cycle of the putative outer companion. At this point we can only confirm the inner planet, and additional observations are required to constrain the orbital properties of GJ 317c.

\section{THE STELLAR MASS-PLANET CORRELATION}

\subsection{Sample Selection}

The mass range encompassed by the California and Carnegie Planet Search (CCPS) target stars provides a large, uniform sample of targets that are ideal for evaluating the effect of stellar mass on the occurrence of Jovian planets. This analysis can be accomplished by simply measuring the fraction of stars with planets in three mass bins: the low-mass $\mathrm{M}$ and late $\mathrm{K}$ dwarfs with $M_{*}<0.7 M_{\odot}$, the Sun-like FGK stars with $0.7 M_{\odot} \leq M_{*}<1.3 M_{\odot}$, and the intermediate-mass subgiants with $1.3 M_{\odot} \leq M_{*} \leq 1.9 M_{\odot}$.

We were careful to select planets that could be reasonably detected around stars with a wide range of masses, and for surveys with different time baselines. Our first requirement is that stars have at least eight observations spanning enough time to search for planets out to a distance of 2.5 AU. Using Kepler's third law, the orbital period scales as $P \propto M_{*}^{-1 / 2}$ for a fixed semimajor axis. An orbital distance of $2.5 \mathrm{AU}$ roughly corresponds to periods

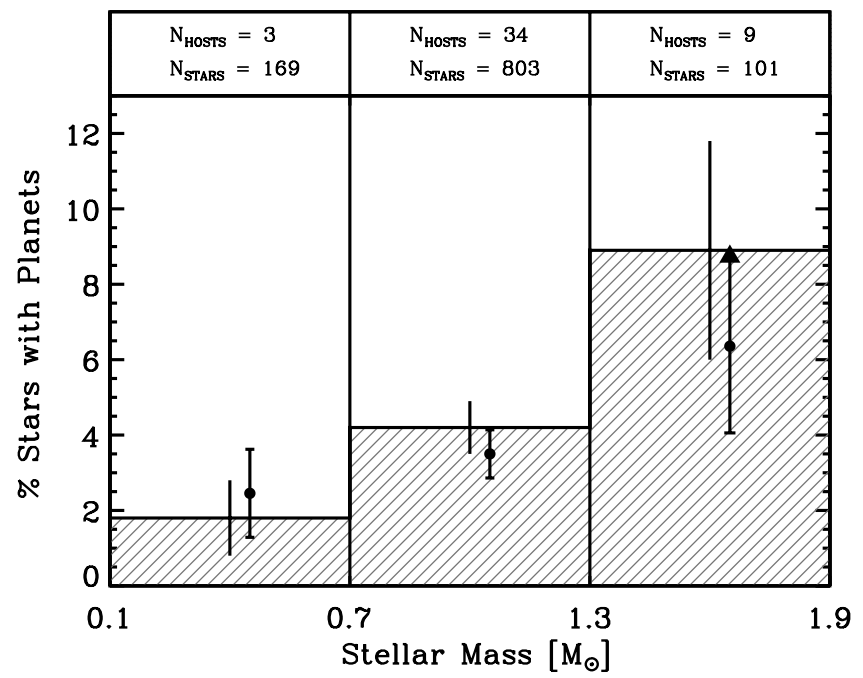

FIG. 6.-Histogram illustrating the rising percentage of stars with detectable planets as a function of stellar mass. The stars selected for each mass bin have eight or more observations providing detectability of planets with masses $M_{P} \sin i \geq$ $0.8 M_{\text {Jup }}$ out to $a=2.5$ AU. After correcting the measured percentages in each mass bin for the effects of stellar metallicity, the rising trend is slightly diminished (circles; offset to the right by $+0.05 M_{\odot}$ for clarity). However, the high-mass bin is uncorrected for the decreased sensitivity of Doppler measurements of higher mass subgiants compared to lower mass stars (cf. $\S 4.3$ ). Our measured occurrence rate for high-mass stars therefore represents a lower limit unlike the solar-mass and low-mass bins. The error bars on each bin are from Poisson statistics, and the numbers above each bin compare the number of stars with planets $N_{\text {HOSTS }}$ to the total number of stars in each bin $N_{\text {STARS }}$.

equal to the 3 year duration of the subgiants planet search, as well as the $\sim 7$ year duration of the NASA Keck M Dwarfs Survey. Next, we exclude stars selected as part of the metallicity-biased Next 2000 Stars (N2K) survey (Fischer et al. 2005). Metallicity is an established tracer of giant planets, and we want to isolate this effect from the effect of stellar mass. We also excluded stars that were added to the CCPS target list after a planet had already been announced by another group.

We included only planet detections that induce a velocity semiamplitude, $K$, large enough to be detected around stars in all three mass bins. For a planet of a given minimum mass and orbital period, the host star's velocity amplitude scales as $K \propto M_{*}^{-2 / 3}$. The larger masses of the subgiants therefore limit the minimum planet mass that can be included in our analysis. Johnson et al. (2007) find that their Doppler measurements of subgiants yield a typical precision of $\sim 6 \mathrm{~m} \mathrm{~s}^{-1}$. We therefore include in our analysis only planets with minimum masses $M_{P} \sin i \geq 0.8 M_{\text {Jup }}$. A planet with this minimum mass in a $P=3$ yr orbit would represent a $2 \sigma$ detection around a $1.6 M_{\odot}$ subgiant, and would be much easier to detect around a lower mass star.

Our choice of such a large minimum planet mass is conservative, since lower mass planets would be readily detectable in shorter orbits. However, restricting our analysis to planets with masses greater than $0.8 M_{\text {Jup }}$ also accomplishes a separate goal. We wish to test the effects of stellar mass on the formation of giant planets. The models of Ida \& Lin (2005a) and Laughlin et al. (2004) suggest that the formation of planets like Jupiter is a threshold process limited by the growth rate of the rocky, embryonic cores. The core growth rate is in turn limited by the supply of solid material at the midplane of the protoplanetary disk, which should scale with the mass of the central star.

\subsection{Jovian Planet Occurrence}

The results of our analysis are displayed in Figure 6 and are summarized here. For the low-mass bin, we selected stars with 
masses $M_{*}<0.7 M_{\odot}$. Of the 147 low-mass stars in the NASA M Dwarf Survey, 130 meet our selection criteria. These stars have masses estimated using the Delfosse et al. (2000) $K$-band massluminosity calibration. We also calculated the masses of all stars with $B-V>1.3$ using the Padova stellar interior models (Girardi et al. 2002). This analysis revealed an additional 39 stars in the mass range $0.6 M_{\odot}<M_{*} \leq 0.7 M_{\odot}$. Of these 169 total stars, only three are known to harbor at least one giant planet: GJ 876, GJ 849, and GJ 317. The resulting planet occurrence rate for these low-mass stars is $1.8 \% \pm 1.0 \%$.

For the solar-mass bin we selected stars from the CCPS Lick, Keck, and Anglo-Australian Observatory surveys with masses in the range $0.7 M_{\odot}<M_{*} \leq 1.3 M_{\odot}$. A total of 803 stars met our stellar mass and planet detectability criteria. Of these stars, 34 harbor at least one giant planet, resulting in a planet occurrence rate of $4.2 \% \pm 0.7 \%$.

For the high-mass bin, we used the intermediate-mass subgiants in the Johnson et al. (2006a) sample, of which 68 meet our selection criteria and have masses in the range $1.3 M_{\odot}<M_{*} \leq$ $1.9 M_{\odot}$, according to the Girardi et al. (2002) stellar interior models. We found an additional 33 intermediate-mass subgiants in the regular CCPS Keck and Lick samples, for a total of 101 stars. There are six published planetary companions among these stars. An additional three companions will be announced in a future publication pending follow-up observations at Lick Observatory in the upcoming observing seasons (J. A. Johnson et al. 2008, in preparation). While these additional planet candidates lack sufficient phase coverage to publish at this time, their signals currently have FAP $<0.01 .^{7}$ Including these strong candidates results in nine giant planets among 101 intermediate-mass subgiants, for an occurrence rate of $8.9 \% \pm 2.9 \%$.

\subsection{The Effects of Metallicity}

The occurrence of detectable planets has previously been shown to correlate with stellar metallicity (Gonzalez 1997; Santos et al. 2004; Fischer \& Valenti 2005). It is therefore important to test whether the correlation between stellar mass and planet occurrence shown in Figure 6 is real, or merely an effect of an underlying metallicity bias.

We searched for such a bias in our sample by measuring the median metallicity in each mass bin. Since the solar-mass stars and intermediate-mass subgiants were analyzed using the same LTE spectral synthesis code (SME), our results from these two mass regimes can be directly compared. For the solar-mass bin we find a median metallicity of $[\mathrm{Fe} / \mathrm{H}]=+0.042$. The median metallicity of the subgiants is slightly higher at $[\mathrm{Fe} / \mathrm{H}]=+0.075$. In order to compare the expected occurrence rate in each of these mass bins based on metallicity alone, we employ the relationship between metallicity and planet probability from Fischer \& Valenti (2005),

$$
p(\text { planet })=0.03 \times 10^{2[\mathrm{Fe} / \mathrm{H}]} .
$$

Given the median metallicity of the solar-mass stars, equation (1) predicts a $3.6 \%$ probability of finding a detectable planet. This is consistent within errors with the $4.2 \% \pm 0.7 \%$ probability that we measure. For the subgiants, the expected probability is $4.2 \%$, which is a factor of 2 lower than the $8.9 \% \pm 2.9 \%$ we measure. This enhanced planet occurrence around our high-mass stars is significant at the $1.6 \sigma$ level.

\footnotetext{
${ }^{7}$ We have analyzed the velocities for our entire sample of $M$ dwarfs and find no candidate signals due to Jovian-mass planets with FAP $<0.01$. Similarly, excluding the metal-rich N2K targets, none of the FGK stars in the CCPS samples has an unpublished candidate with $M_{P} \sin i \geq 0.8 M_{\text {Jup }}$ within $2.5 \mathrm{AU}$.
}

The correlation between stellar mass and exoplanets in Figure 6 is most apparent when comparing the bins at either mass extreme. The high-mass stars in our sample appear to be planetenriched by a factor of 5 compared to the low-mass $\mathrm{M}$ dwarfs, which represents a $2.3 \sigma$ result. However, it is currently very difficult to derive spectroscopic abundances for M-type stars. ${ }^{8}$ Could it be that there is a systematic metallicity bias among our low-mass stars?

A common argument for such a metallicity bias among $\mathrm{M}$ dwarfs is related to their age (e.g., Bonfils et al. 2005b). According to this argument, $\mathrm{M}$ dwarfs have lifetimes longer than the age of the Galaxy and are therefore more likely than higher mass stars to have formed in the distant past when the Galaxy was metal-poor. This argument is based on the assumption that there is a welldefined age-metallicity relationship in the Galaxy. However, studies of Galactic chemical evolution have shown that no well-defined correlation between age and metallicity exists in the thin Galactic disk. Analyses of the local stellar population show that stars have a wide range of abundances at ages from 1 to $10 \mathrm{Gyr}$, with a $0.2 \mathrm{dex}$ scatter in $[\mathrm{Fe} / \mathrm{H}]$ over this age range (Edvardsson et al. 1993; Nordström et al. 2004; Takeda et al. 2007). Similarly, open clusters with comparable ages also exhibit a wide range of metallicities, with 3-6 Gyr clusters differing by as much as 0.82 dex (e.g., Chen et al. 2003). Furthermore, late G- and all K-type stars also have lifetimes comparable to the age of the Galaxy. Therefore, any metallicity bias present in our sample of $\mathrm{M}$ dwarfs should also be present in a volume-limited sample of $\mathrm{G}$ and $\mathrm{K}$ dwarfs. We therefore find no compelling reason to suspect that the abundances of $\mathrm{M}$ dwarfs should deviate significantly from the $\mathrm{G}$ and $\mathrm{K}$ stars in the solar neighborhood.

Our sample of M dwarfs within $15 \mathrm{pc}$ of the Sun should trace the metal abundances of the other stars in the immediate solar neighborhood. The volume-limited sample of Fischer \& Valenti (2005) has a mean metallicity of $[\mathrm{Fe} / \mathrm{H}]=-0.05$. This is roughly the abundance we expect for our sample of $\mathrm{M}$ dwarfs. We stress that, contrary to the argument above, our sample of $\mathrm{M}$ dwarfs is not metal-poor compared to other nearby stars. Rather it is the solar-mass stars and subgiants among the planet search targets that are slightly metal-rich compared to a volume-limited sample. This effect has been previously noted by Fischer \& Valenti (2005) and is related to the fact that in a given range of $B-V$ colors metalrich stars are brighter and more likely to be included in the samples of most Doppler-based planet searches (see also Marcy et al. [2005] for a more detailed discussion).

Based on our estimate for the mean metallicity of our sample of $\mathrm{M}$ dwarfs $([\mathrm{Fe} / \mathrm{H}]=-0.05)$, equation (1) predicts a $2.4 \%$ planet occurrence rate for the stars in our low-mass bin. This prediction is slightly higher than our measured occurrence rate of $1.8 \% \pm 1.0 \%$, but the two values agree within errors.

We can use the probabilities predicted by equation (1) to correct our measured occurrence rates for the effects of stellar metallicity. Each corrected planet fraction, $f_{i}^{\prime}$, is constructed as

$$
f_{i}^{\prime}=\left(p_{\odot} / p_{i}\right) f_{i},
$$

where $f_{i}$ is the original planet fraction, $p_{\odot}=0.03$ is the expected probability for solar metallicity, and $p_{i}$ is the predicted probability from equation (1). Similarly, the corrected uncertainty in each bin is

$$
\sigma_{i}^{\prime}=\sqrt{p_{\odot} / p_{i}} \sigma_{i}
$$

\footnotetext{
${ }^{8}$ See, however, Bean et al. (2006) for spectroscopically derived metallicities of three $\mathrm{M}$ dwarf planet host stars.
} 
The circles in Figure 6 show the corrected values and uncertainties of the planet occurrence rate in each mass bin. After correcting for metallicity, the contrast between the low-mass and high-mass bins is diminished. However, evidence for an increasing trend remains: the A-type stars have a factor of 2.5 higher planet occurrence rate than the $\mathrm{M}$ dwarfs.

It is important to note that the corrected occurrence rates in Figure 6 do not account for the decreased sensitivity of our Doppler survey of subgiants compared to the surveys of lower mass stars. Our requirement of eight or more observations was exceeded in most cases by the stars in the solar-mass and low-mass bins. The solar-mass stars had on average 21 observations each, and the M dwarfs averaged 16 observations. On the other hand, the subgiants had an average of only 10 observations each. Also, whereas our typical Doppler precision is $\leqslant 2 \mathrm{~m} \mathrm{~s}^{-1}$ for solar-mass stars (Marcy et al. 2005; Johnson et al. 2006b) and 3-4 $\mathrm{m} \mathrm{s}^{-1}$ for M dwarfs (Fig. 2), the subgiants typically have a precision of 4$7 \mathrm{~m} \mathrm{~s}^{-1}$ due to their larger jitter (Johnson et al. 2007). The sensitivity of our measurements of subgiants is further diminished, since the amplitude of a star's reflex motion caused by a planet with a given mass and orbital period scales as $K \propto M_{*}^{-2 / 3}$. We therefore conclude that the measured planet occurrence rate for the stars in our high-mass bin represents a lower limit rather than an absolute measurement of the true fraction of subgiants with planets (as indicated by the upward-pointing arrow in Fig. 6), even after correcting for metallicity.

\section{SUMMARY AND DISCUSSION}

We present the detection of a Jupiter-mass planet orbiting the M3.5 dwarf GJ 317. This detection marks the sixth M dwarf known to harbor at least one Doppler-detected planet, together with GJ 876 (Marcy et al. 1998, 2001; Delfosse et al. 1998; Rivera et al. 2005), GJ 436 (Butler et al. 2004), GJ 581 (Bonfils et al. 2005b; Udry et al. 2007), GJ 849 (Butler et al. 2006a), and GJ 674 (Bonfils et al. 2007). GJ 317 is only the third M dwarf with a Doppler-detected, Jupiter-mass planet. In addition to our firm detection of a planet with $P=692.9$ days, we also detect evidence of a possible second Jovian planet in the system near $P \approx 2700$ days (Fig. 4). However, additional monitoring is required to fully characterize the orbit of the second planet.

Multiplanet systems appear to be relatively common among M dwarfs compared to Sun-like stars. All M stars with one Jovian planet show evidence of a second companion. GJ 876 has a pair of Jupiter-mass planets in a 2:1 mean motion resonance, along with an inner super-Earth (Marcy et al. 2001; Rivera et al. 2005). GJ 849 has a long-period Jovian planet with a linear trend (Butler et al. 2006a). Of the three M dwarfs with Neptune-mass planets, two have multiple planets or evidence of an additional companion: GJ 581 harbors three low-mass planets (Udry et al. 2007), and GJ 436 has a linear trend (Maness et al. 2007). Only GJ 674 appears to be in a single-planet system. From the first six planet detections around low-mass stars, it appears as though M dwarfs have an $80 \%$ occurrence rate of multiplanet systems, compared to the $30 \%$ rate measured for FGK stars (Wright et al. 2007).

The high frequency of multicomponent detections around M dwarfs may be in part due to the increased detectability of planets around low-mass stars. This is likely the case for the triple Neptune system around GJ 581. The "c" component in that system would have induced a velocity amplitude of only $1.4 \mathrm{~m} \mathrm{~s}^{-1}$ if it orbited a $1 M_{\odot}$ star. However, the enhanced detectability of planets around $\mathrm{M}$ stars should not matter as much for the systems containing Jovian planets, since all of the Jovian planets orbiting $\mathrm{M}$ stars would be detectable around solar-mass stars.
We studied the relationship between stellar mass and the occurrence rate of giant planets by combining our sample of $\mathrm{M}$ dwarfs with our samples of solar-mass FGK stars and intermediate-mass subgiants. By measuring the fraction of stars with planets in three stellar mass bins we find that the frequency of planets with $M_{*}>$ $0.8 M_{\text {Jup }}$ within $a<2.5 \mathrm{AU}$ increases with stellar mass (Fig. 6). The evolved A-type stars in our sample are nearly 5 times more likely than $\mathrm{M}$ dwarfs to harbor a giant planet. This important result establishes stellar mass as an additional signpost for exoplanets, along with metallicity. Just as metallicity informs the target selection of searches for short-period planets (e.g., Fischer et al. 2005), stellar mass will be an important factor in the target selection of future high-contrast direct imaging surveys. For example, while the lower luminosities of $\mathrm{M}$ dwarfs provide favorable contrast ratios that facilitate the detection of thermal emission from young giant planets, our results show that A-type stars are far more likely to harbor such planets.

In order to understand the role of stellar mass on planet formation, it is important to disentangle the known effects of stellar metallicity from our stellar sample. After correcting for metallicity in each mass bin, the slope of the trend in Figure 6 is slightly diminished. However, the factor of 2.5 increase in planet occurrence around A stars compared to $\mathrm{M}$ dwarfs remains significant, especially considering that the high-mass bin is uncorrected for the relatively lower detection sensitivity of the subgiants planet search. Our results therefore confirm the prediction of the core accretion model that the efficiency of Jovian planet formation should increase with stellar mass (Laughlin et al. 2004; Ida \& Lin 2005b; Kennedy \& Kenyon 2007).

Our discovery confirms and expands on the recent results of Lovis \& Mayor (2007), who are searching planets around intermediate-mass K giants. By focusing on stars in open clusters, they are able to control for the age and metallicity of their sample and determine accurate stellar masses. Because of the larger stellar radii and lower precision (jitter $>15 \mathrm{~m} \mathrm{~s}^{-1}$ ) of $\mathrm{K}$ giants, they focused on planets with $M_{P} \sin i \geq 5 M_{\text {Jup }}$ and $0.5 \mathrm{AU} \leq a \leq$ 2.5 AU. Lovis \& Mayor (2007) find that the occurrence rate of "super-Jupiters" and brown dwarfs within this mass and semimajor axis range increases with stellar mass, rising from $0 \%$ for $\mathrm{M}$ dwarfs to $2.5 \%$ for evolved A stars.

The high-mass sample of stars analyzed by Lovis \& Mayor (2007) contained primarily stars with masses $M_{*} \gtrsim 2.0 M_{\odot}$. In order to avoid confusion with solar-mass, horizontal-branch stars ("clump giants") we have restricted our subgiants planet search primarily to stars with absolute magnitudes $M_{V}>1.8$, corresponding to masses $M_{*} \lesssim 2.1 M_{\odot}$ (Johnson et al. 2006a). Our planet search is therefore complementary to the survey of Lovis \& Mayor (2007) and other planet searches around K giants (e.g., Setiawan et al. 2003; Sato et al. 2003; Hatzes et al. 2005; Reffert et al. 2006; Niedzielski et al. 2007). The $\mathrm{K}$ giants provide information about massive planets around stars with $M_{*} \gtrsim 2.1 M_{\odot}$, and the relatively stable atmospheres of subgiants ( jitter $=5 \mathrm{~m} \mathrm{~s}^{-1}$ ) allow us to detect planets beyond $1 \mathrm{AU}$ with minimum masses down to $M_{P} \sin i \approx 0.5 M_{\text {Jup }}$.

Our preliminary results and those of Lovis \& Mayor (2007) reveal a rough, positive correlation between the mass of stars and the likelihood that they harbor giant planets. However, testing the exact shape of the relationship predicted by the simulations of Kennedy \& Kenyon (2007) - a rising trend of planet occurrence up to a peak of $\approx 20 \%$ near $3 M_{\odot}$-will require a much larger sample of stars. We have expanded our search for planets around intermediate-mass stars by adding 300 additional evolved A and $\mathrm{F}$ stars to our Lick and Keck samples. The results from our 
expanded planet search should reduce the error bar on the highmass bin in Figure 6 by a factor of 2 . If the $9 \%$ occurrence rate for $M_{*}>1.3 M_{\odot}$ is confirmed, then our expanded planet search will result in the detection of 20-30 new planets orbiting some of the most massive planet host stars in the solar neighborhood. In addition to verifying the preliminary results presented here, these planet detections will also allow us to study the effects of stellar mass on other planet characteristics such as eccentricity, semimajor axis, and minimum mass.

We gratefully acknowledge the efforts and dedication of the Lick Observatory and Keck Observatory staff. We are also grateful to the time assignment committees of NASA, NOAO, and the University of California for their generous allocations of observing time. We thank Ben Zuckerman for his thought-provoking conversations regarding $\mathrm{M}$ dwarf metallicities. We acknowledge support by NSF grants AST 07-02821 (to J. A. J.) and AST 03-07493 (to S. S. V.); AST 99-88087, NASA grant NAG5-12182, and travel support from the Carnegie Institution of Washington (to R. P. B.); and NASA grant NAG5-8299 and NSF grant AST 9520443 (to G. W. M.). D. A. F. is a Cottrell Science Scholar of Research Corporation and acknowledges support from NASA grant NNG 05G164G, which made this work possible. This research has made use of the Simbad database operated at CDS, Strasbourg, France, and the NASA ADS database. Finally the authors wish to extend thanks to those of Hawaiian ancestry on whose sacred mountain of Mauna Kea we are privileged to be guests. Without their generous hospitality, the Keck observations presented herein would not have been possible.
Bean, J. L., Benedict, G. F., \& Endl, M. 2006, ApJ, 653, L65

Beaulieu, J.-P., et al. 2006, Nature, 439, 437

Bennett, D. P., Anderson, J., Bond, I. A., Udalski, A., \& Gould, A. 2006, ApJ, 647, L171

Bond, I. A., et al. 2004, ApJ, 606, L155

Bonfils, X., Delfosse, X., Udry, S., Santos, N. C., Forveille, T., \& Ségransan, D. 2005a, A\&A, 442, 635

Bonfils, X., et al. 2005b, A\&A, 443, L15 2007, A\&A, 474, 293

Butler, R. P., Johnson, J. A., Marcy, G. W., Wright, J. T., Vogt, S. S., \& Fischer, D. A. 2006a, PASP, 118, 1685

Butler, R. P., Marcy, G. W., Williams, E., McCarthy, C., Dosanjh, P., \& Vogt, S. S. 1996, PASP, 108, 500

Butler, R. P., Vogt, S. S., Marcy, G. W., Fischer, D. A., Wright, J. T., Henry, G. W., Laughlin, G., \& Lissauer, J. J. 2004, ApJ, 617, 580

Butler, R. P., et al. 2006b, ApJ, 646, 505

Chen, L., Hou, J. L., \& Wang, J. J. 2003, AJ, 125, 1397

Delfosse, X., Forveille, T., Mayor, M., Perrier, C., Naef, D., \& Queloz, D. 1998, A\&A, 338, L67

Delfosse, X., Forveille, T., Ségransan, D., Beuzit, J.-L., Udry, S., Perrier, C., \& Mayor, M. 2000, A\&A, 364, 217

do Nascimento, J. D., Canto Martins, B. L., Melo, C. H. F., Porto de Mello, G., \& De Medeiros, J. R. 2003, A\&A, 405, 723

Edvardsson, B., Andersen, J., Gustafsson, B., Lambert, D. L., Nissen, P. E., \& Tomkin, J. 1993, A\&A, 275, 101

Endl, M., Cochran, W. D., Kürster, M., Paulson, D. B., Wittenmyer, R. A., MacQueen, P. J., \& Tull, R. G. 2006, ApJ, 649, 436

Endl, M., Cochran, W. D., Tull, R. G., \& MacQueen, P. J. 2003, AJ, 126, 3099

Fischer, D. A., \& Valenti, J. 2005, ApJ, 622, 1102

Fischer, D. A., et al. 2005, ApJ, 620, 481

Fortney, J. J., Marley, M. S., \& Barnes, J. W. 2007, ApJ, 659, 1661

Galland, F., Lagrange, A.-M., Udry, S., Chelli, A., Pepe, F., Queloz, D., Beuzit, J.-L., \& Mayor, M. 2005, A\&A, 443, 337

Gillon, M., et al. 2007, A\&A, 472, L13

Girardi, L., Bertelli, G., Bressan, A., Chiosi, C., Groenewegen, M. A. T., Marigo, P., Salasnich, B., \& Weiss, A. 2002, A\&A, 391, 195

Gliese, W., \& Jahreiss, H. 1991, Preliminary Version of the Third Catalogue of Nearby Stars (Greenbelt: NASA)

Gonzalez, G. 1997, MNRAS, 285, 403

Gould, A., et al. 2006, ApJ, 644, L37

Hatzes, A. P., Guenther, E. W., Endl, M., Cochran, W. D., Döllinger, M. P., \& Bedalov, A. 2005, A\&A, 437, 743

Ida, S., \& Lin, D. N. C. 2004, ApJ, 616, 567

\section{REFERENCES}

Ida, S., \& Lin, D. N. C. 2005a, Prog. Theor. Phys. Suppl., 158, 68 2005b, ApJ, 626, 1045

Johnson, J. A., Marcy, G. W., Fischer, D. A., Henry, G. W., Wright, J. T.,

Isaacson, H., \& McCarthy, C. 2006a, ApJ, 652, 1724

Johnson, J. A., et al. 2006b, ApJ, 647, 600 2007, ApJ, 665, 785

Kennedy, G. M., \& Kenyon, S. J. 2007, ApJ, in press (arXiv: 0710.1065)

Kornet, K., Bodenheimer, P., Różyczka, M., \& Stepinski, T. F. 2005, A\&A, 430,1133

Laughlin, G., Bodenheimer, P., \& Adams, F. C. 2004, ApJ, 612, L73

Laws, C., Gonzalez, G., Walker, K. M., Tyagi, S., Dodsworth, J., Snider, K., \& Suntzeff, N. B. 2003, AJ, 125, 2664

Lovis, C., \& Mayor, M. 2007, A\&A, 472, 657

Maness, H. L., Marcy, G. W., Ford, E. B., Hauschildt, P. H., Shreve, A. T.,

Basri, G. B., Butler, R. P., \& Vogt, S. S. 2007, PASP, 119, 90

Marcy, G. W., \& Butler, R. P. 1992, PASP, 104, 270

Marcy, G. W., Butler, R. P., Fischer, D., Vogt, S. S., Lissauer, J. J., \& Rivera, E. J. 2001, ApJ, 556, 296

Marcy, G. W., Butler, R. P., Vogt, S. S., Fischer, D. A., Henry, G. W., Laughlin, G., Wright, J. T., \& Johnson, J. A. 2005, ApJ, 619, 570

Marcy, G. W., Butler, R. P., Vogt, S. S., Fischer, D., \& Lissauer, J. J. 1998, ApJ, 505, L147

Niedzielski, A., et al. 2007, ApJ, 669, 1354

Nordström, B., et al. 2004, A\&A, 418, 989

Rauscher, E., \& Marcy, G. W. 2006, PASP, 118, 617

Reffert, S., Quirrenbach, A., Mitchell, D. S., Albrecht, S., Hekker, S., Fischer,

D. A., Marcy, G. W., \& Butler, R. P. 2006, ApJ, 652, 661

Reid, I. N., Kilkenny, D., \& Cruz, K. L. 2002, AJ, 123, 2822

Rivera, E. J., et al. 2005, ApJ, 634, 625

Saar, S. H., Butler, R. P., \& Marcy, G. W. 1998, ApJ, 498, L153

Santos, N. C., Israelian, G., \& Mayor, M. 2004, A\&A, 415, 1153

Sato, B., et al. 2003, ApJ, 597, L157

Setiawan, J., et al. 2003, A\&A, 398, L19

Takeda, G., Ford, E. B., Sills, A., Rasio, F. A., Fischer, D. A., \& Valenti, J. A. 2007, ApJS, 168, 297

Udry, S., et al. 2007, A\&A, 469, L43

Valenti, J. A., Butler, R. P., \& Marcy, G. W. 1995, PASP, 107, 966

Valenti, J. A., \& Fischer, D. A. 2005, ApJS, 159, 141

Vogt, S. S., et al. 1994, Proc. SPIE, 2198, 362

Woolley, R. v. d. R. 1970, R. Obs. Ann., 5

Wright, J. T. 2004, AJ, 128, 1273 2005, PASP, 117, 657

Wright, J. T., et al. 2007, ApJ, 657, 533 